\title{
ANÁLISE DO PERFIL DO ESTILO DE VIDA DE POLICIAIS MILITARES DO ESTADO DO PARANÁ
}

\section{ANALYSIS OF THE LIFESTYLE PROFILE OF MILITARY POLICE IN THE STATE OF PARANA}

José Roberto Andrade do Nascimento Júnior, Leidyane Aparecida de Souza Amoroso de Souza, Gislaine Contessoto Pizzo, Daniel Vicentini de Oliveira, Lenamar Fiorese Vieira

\section{RESUMO}

O presente estudo teve como objetivo analisar o perfil do estilo de vida de policiais militares do estado do Paraná do sexo masculino e feminino. Estudo transversal, realizado com 144 policiais militares. Como instrumento foi utilizado o questionário "Perfil do Estilo de Vida", que avalia os componentes do estilo de vida por meio do Pentáculo do Bem-estar (nutrição, atividade física, comportamento preventivo, relacionamentos e controle de estresse). Os resultados evidenciaram que as mulheres apresentaram melhor estilo de vida nos componentes de nutrição, atividade física, relacionamentos e controle de estresse em comparação aos homens. Verificou-se também correlações positivas da atividade física com a nutrição e o controle do estresse para ambos os sexos. Conclui-se que as mulheres possuem estilo de vida mais saudável em comparação aos homens, além da importância da atividade física para ambos os sexos, uma vez que este componente esteve relacionado com a nutrição e controle do estresse.

Descritores: Polícia Milita; Estilo de vida; Saúde.

\section{ABSTRACT}

The present study had the objective of analyzing the lifestyle profile of military policemen of the state of Paraná, male and female. A cross-sectional study of 144 military police officers. As a tool, the "Lifestyle Profile" questionnaire assesses lifestyle components through the Welfare Pentacle (nutrition, physical activity, preventive behavior, relationships, and stress management). The results showed that women presented better lifestyle in the components of nutrition, physical activity, relationships and stress control compared to men. There were also positive correlations of physical activity with nutrition and stress control for both sexes. It is concluded that women have a healthier lifestyle compared to men, besides the importance of physical activity for both sexes, since this component was related to nutrition and stress control.

Descriptors: Police; Lifestyle; Health.

José Roberto Andrade do Nascimento Júnior, Doutor em Educação fisica pela Universidade Estadual de Maringá (UEM), Maringá, Paraná, Brasil.

Leidyane Aparecida de Souza Amoroso de Souza, Graduada em Educação fisica pela Faculdade Metropolitana de Maringá (FAMMA), Maringá, Paraná, Brasil

Gislaine Contessoto Pizzo, Mestre em Educação física pela Universidade Estadual de Maringá (UEM), Maringá, Paraná, Brasil.

Daniel Vicentini de Oliveira, Doutorando em Gerontologia pela Universidade Estadual de Campinas (UNICAMP), Campinas, SP, Brasil.

Lenamar Fiorese Vieira, Doutora em Ciência do Movimento Humano pela Universidade Federal de Santa Maria (UFSM), Santa Maria, Rio Grande do Sul, Brasil. 
Introdução

A atuação profissional tem sido apontada como um fator interveniente no estilo de vida do indivíduo, uma vez que 0 trabalho é caracterizado como uma das principais atividades desenvolvidas pelo homem1. Neste sentido, o exercício da profissão e sua sobrecarga pode provocar nos trabalhadores desgastes físicos e emocionais, o que pode refletir em prejuízos a saúde e à qualidade de vida2. Uma das profissões que está entre as categorias profissionais que se expõe a riscos e tem sua integridade a saúde ameaçada é o trabalho de policial, por conta de fatores que influenciam o seu desempenho nessa profissão, como o risco de morte, convivência com a violência e contato com problemas da sociedade3.

Dentro do serviço policial militar, existem vários tipos de jornada de trabalho, as quais são consideradas desgastantes $\mathrm{e}$ estressantes. Neste sentido destaca-se que é trabalhando que o policial passa a maior parte de sua vida; é o trabalho, ou por meio dele, que é realizado grande parte das aspirações e é daí que vem a relevância do papel do trabalho na vida e o reflexo do restante dela sobre a qualidade do trabalho e o desempenho profissional4.

Para o desempenho de suas funções, o policial militar precisa estar disposto para atender às novas e crescentes exigências que as questões de segurança pública Ihe demandam5. De modo peculiar, deve ser munido de recursos técnicos e táticos para intervir em favor da ordem social6. Dessa forma, o policial militar deve gozar de boa saúde, aptidão física acima da média populacional, ter o nível de estresse controlado, e possuir qualidades físicas como: a força agilidade, resistência aeróbica e anaeróbica e coordenação motora7. Por isso se faz necessário um bom nível de aptidão física para o desempenho do serviço policial militar, no cumprimento do dever constitucional de preservar a ordem pública e executar o policiamento ostensivo8.

Neste sentindo, pesquisas têm sido realizadas com o objetivo de investigar aspectos relacionados à qualidade de vida de policiais, associadas a níveis de estresse, prática de atividade física, sofrimento psíquico e saúde mental,9-12,3,1. № entanto nota-se uma lacuna na literatura a respeito de estudos que investigam a percepção de estilo de vida em policiais com base em um modelo teórico específico.

No entanto, no presente estudo o estilo de vida será investigado com base no modelo do Pentáculo do bem-estar proposto por Nahas 13. 0 modelo inclui aspectos fundamentais do estilo de vida das pessoas que afetam a saúde geral e estão associados ao bem-estar psicológico e às diversas doenças crônico-degenerativas. Este modelo possui cinco componentes, tais como: o componente nutrição, que está relacionado à hábitos alimentares; o componente preventivo, que se diz respeito a cuidados com a saúde; o componente relacionamento social, que se refere aos momentos de lazer encontro com amigos e participações em associações; e o componente controle do estresse que está direcionado à maneira que a pessoa se controla, a estratégias que utilizam diante de eventos que causam estresse 14

Frente ao exposto, entende-se que para cumprimento da missão diária no trabalho é necessário que haja um equilíbrio entre bem-estar físico, mental e social do policial militar e ainda tenha um bom convívio familiar, boas condições de trabalho, mantenha a prática regular de atividades físicas, uma alimentação equilibrada e que desfrute de momentos de lazer no âmbito familiar15. Uma vez que a qualidade de vida é definida como a condição humana resultante de um conjunto de parâmetros individuais e socioambientais, modificáveis ou não, que caracterizam as condições em que vive o ser humano16. Dentre os parâmetros individuais, o estilo de vida é um dos importantes determinantes da saúde de indivíduos, grupos e comunidades. Enquanto nos parâmetros socioambientais destacam-se as condições de trabalho, remuneração, educação e lazer, dentre outros16. No mesmo sentido, Kloster e Prati17, reforçam que a saúde do ser humano é extremamente influenciada pelo estilo de vida que possui, uma vez que, é construído a partir da maneira diária de se alimentar, se investir no autoconhecimento, nos relacionamentos interpessoais, na prática de atividades físicas, e na não adoção de hábitos nocivos à saúde16.

Diante do exposto, o presente estudo tem por objetivo analisar o perfil do estilo de vida de policiais do $4^{\circ}$ Batalhão da Polícia Militar do município de Maringá no estado do Paraná, buscando especificamente comparar o perfil do estilo de vida dos policiais do sexo masculino e feminino e verificar a correlação entre os componentes de estilo de vida.

Metodologia

População e amostra

Foram convidados a participar do estudo todos os policiais do $4^{\circ}$ Batalhão da Polícia Militar do município de
Maringá no estado do Paraná, totalizando um efetivo de 605 policiais. No entanto, apenas 144 policiais aceitaram voluntariamente fazer parte da pesquisa, sendo 92 policiais do sexo masculino (média de idade de $36,33 \pm 8,65$ anos) $e$ 52 do sexo feminino (média de idade de 32,02 $\pm 8,29$ anos), os quais estavam empregados em trabalhos operacionais e administrativos.

Instrumento de medida

O instrumento utilizado neste estudo foi o questionário de Nahas, Barros e Francalacci13, intitulado "Perfil do Estilo de Vida", que traz como componentes do estilo de vida a Nutrição, a Atividade Física, o Comportamento Preventivo, os Relacionamentos e o Controle de Estresse. Cada um desses componentes é avaliado por 3 questões, as quais teriam como opção de resposta as afirmativas: nunca, às vezes, quase sempre e sempre, correspondente a valores de $0,1,2$ e 3, respectivamente. Após a classificação citada, fez-se, para cada componente do instrumento (são 5), uma média dos pontos marcados $(0,1,2,3)$. Assim, os sujeitos obtiveram um escore de 0 a 3 pontos em cada um dos cinco componentes do perfil do estilo de vida.

\section{Coleta de dados}

O estudo foi submetido ao comitê de ética da Universidade Estadual de Maringá, sob parecer aprovado $\mathrm{n}^{0}$ 238/2011. Para fins de coleta de dados, foi realizado um contato com o comandante do $4^{\circ}$ Batalhão da Polícia Militar da cidade de Maringá, solicitando autorização para realizar uma coleta de dados com os policiais do batalhão. Mediante sua autorização, os policiais que aceitaram participar da pesquisa assinaram o termo de consentimento livre e esclarecido. Os policiais foram reunidos conforme suas disponibilidades, para explicação e aplicação do questionário.

\section{Análise de dados}

Para a análise dos dados, foi verificada a normalidade dos dados por meio do teste Kolmogorov-Smirnov. Como os dados não apresentaram distribuição normal, foram utilizadas Mediana (Md) e Quartis (Q1; Q3) para a caracterização dos resultados. Como se pretendia verificar as maiores percepç̃es em relação aos componentes do estilo de vida dos sujeitos de uma forma geral, foi utilizada a Anova de Medidas Repetidas, após a verificação da esfericidade dos dados, seguida do Post Hoc de Bonferroni corrigido $(p<0,01$ ). Na comparação entre os grupos (masculino e feminino), foi utilizado o teste "U" de Mann-Whitney. Para verificar a correlação entre as variáveis, efetuou-se o coeficiente de correlação de Spearman. A significância adota foi de $p<0,05$. Os dados foram analisados no software versão SPSS 22.0.

RESULTADOS:

Nota-se (Tabela 1) que, de uma forma geral, os componentes de estilo de vida que mais prevaleceram entre os policiais do $4^{\circ}$ Batalhão do Paraná foram o Comportamento Preventivo $(M d=2,00)$ e Relacionamento Social $(M d=$ $1,67)$, visto que apresentaram diferença significativa $(p<0,01)$ com o componente que obteve menor valor, o Controle do Estresse $(M d=1,00)$.

Tabela 1 - Comparação dos componentes do perfil de estilo de vida predominantes dos policiais do $4^{\circ}$ Batalhão da Polícia Militar do Paraná

\begin{tabular}{lr}
\hline Domínios de Estilo de vida & $\mathrm{Md}(\mathrm{Q} 1 ; \mathrm{Q} 3)$ \\
Nutricional & $1,27(0,67 ; 2,00) \mathrm{a} / \mathrm{b}$ \\
Atividade Física & $1,50(0,67 ; 2,00) \mathrm{c} / \mathrm{d}$ \\
Comportamento Preventivo & $2,00(1,66 ; 2,33) \mathrm{a} / \mathrm{c} / \mathrm{e} / \mathrm{f}$ \\
Relacionamento Social & $1,67(1,00 ; 2,00) \mathrm{b} / \mathrm{e} / \mathrm{g}$ \\
Controle de Estresse & $1,00(0,67 ; 1,67) \mathrm{d} / \mathrm{f} / \mathrm{g}$
\end{tabular}

* Diferença significativa: $p<0,01$ entre: a) Nutricional e Comp. Preventivo; b) Nutricional e Relacionamento 
Social; c) Atividade Física e Comp. Preventivo; d) Atividade Física e Controle do Estresse; e) Comp. רา-Preventivo e Relacionamento Social; f) Comp. Preventivo e Controle do Estresse; g) Relacionamento Social e Controle do Estresse.

Ao comparar os domínios do perfil de estilo de vida dos policiais do sexo masculino e feminino do $4^{\circ}$ Batalhão da Polícia Militar do Paraná (Tabela 2), foi encontrada diferença significativa $(p<0,05)$ entre os grupos em todos os componentes, com exceção do componente Comportamento Preventivo $(p=0,059)$.

Tabela 2 - Comparação dos componentes do perfil de estilo de vida dos policiais do sexo masculino e feminino do $4^{\circ}$ Batalhão da Polícia Militar do Paraná.

\begin{tabular}{lccl}
\hline Domínios de Estilo de vida & $\begin{array}{c}\text { Masculino }(n=92) \\
M d(Q 1 ; Q 3)\end{array}$ & $\begin{array}{c}\text { Feminino }(n=52) \\
M d(Q 1 ; Q 3)\end{array}$ & $p$ \\
\hline Nutricional & $1,00(0,33 ; 1,67)$ & $1,67(1,08 ; 2,25)$ & $0,001^{*}$ \\
Atividade Física & $1,00(0,33 ; 1,67)$ & $1,67(1,42 ; 2,33)$ & $0,001^{*}$ \\
Comportamento Preventivo & $2,00(1,67 ; 2,33)$ & $2,17(1,67 ; 2,67)$ & 0,059 \\
Relacionamento Social & $1,33(0,67 ; 2,00)$ & $2,00(1,67 ; 2,33)$ & $0,001^{*}$ \\
Controle de Estresse & $1,00(0,42 ; 1,33)$ & $1,50(1,00 ; 2,00)$ & $0,001^{*}$ \\
\hline
\end{tabular}

" Diferença significativa: $p<0,05-$ Teste "U" de Mann-Whitney.

Ao analisar a correlação entre os componentes de estilo de vida nas mulheres, destacam-se correlações significativas $(p<0,05)$ e positivas entre os seguintes componentes: Nutricional e Atividade física $(r=0,509)$; Atividade física e Comportamento Preventivo ( $r=0,325)$; Atividade física e Controle de Estresse $(r=0,352)$.

Tabela 3 - Correlação entre os componentes do perfil de estilo de vida dos policiais do sexo masculino e feminino do $4^{\circ}$ Batalhão da Polícia Militar do Paraná.

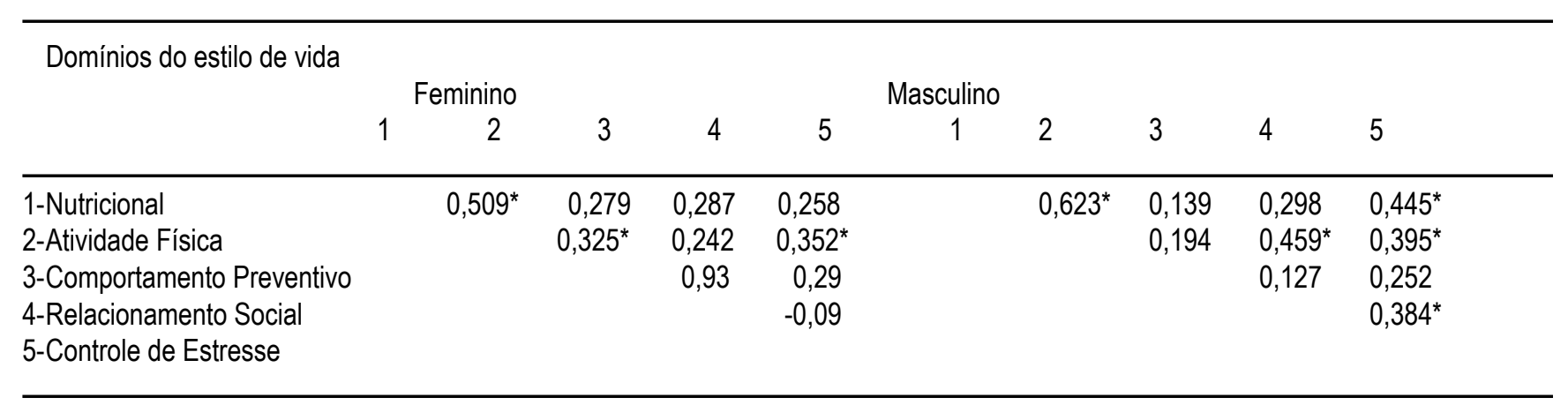

${ }^{*}$ Correlação Significativa $-p<0,05$. Correlação de Spearman.

Já para os homens (Tabela 3), foram encontradas correlações significativas $(p<0,05)$ e positivas entre a dimensão nutricional com Atividade física ( $r=0,623$ ); nutricional e Controle de Estresse ( $r=0,445)$; Atividade Física e Relacionamento Social $(r=0,459)$; Atividade Física e Controle de Estresse $(r=0,395)$ e Relacionamento Social e Controle de Estresse $(\mathrm{r}=0,384)$.

\section{Discussão}

Em busca de preencher a lacuna encontrada na literatura, o presente estudo objetivou analisar o perfil de estilo de vida dos policiais do $4^{\circ}$ Batalhão da Polícia Militar do Paraná, com base no modelo do Pentáculo do Bem-Estar que inclui cinco aspectos fundamentais do estilo de vida das pessoas e que sabidamente afetam a saúde geral e estão associados ao bem-estar psicológicos e a diversas doenças crônico-degenerativas, como infarto do miocárdio, o derrame cerebral, 0 diabetes, a hipertensão, a obesidade e a osteoporose18.
Os resultados encontrados no estudo (Tabela 1) indicam que os policiais participantes da pesquisa, caracterizamse como indivíduos que predominantemente adotam um comportamento preventivo relacionado a saúde, procuram manter um bom relacionamento social, e praticam atividade física. No modelo proposto por Nahas13, ressalta-se a importância de se conservar hábitos preventivos, uma vez que são comportamentos relacionados à saúde, que passaram a ser fundamentais na vida humana. Estes comportamentos estão relacionados a uso de equipamentos de segurança no trabalho, ingestão moderada de bebida alcoólicas, não usar drogas entre outros. Infere-se que policiais adotam esses comportamentos preventivos uma vez que sua profissão exige os referidos cuidados19. Os resultados observados no componente Relacionamento Social indicam que os policiais militares mantem um relacionamento social positivo. O relacionamento social é um importante item que compõe a qualidade de vida, e é entendido com uma válvula de escape para o estresse, pois o sentimento de isolamento aliado ao acumulo de estresse, pode desencadear doenças cardíacas e estresse crônico4. Entende-se assim que o impacto das relações sociais da pessoa, em especial sobre seu relacionamento com a família, no trabalho e no lazer, realização de expectativas e satisfação com a vida sexual, além dos sentimentos de acesso a bons cuidados médicos, amparo da sociedade e apoio dos amigos e familiares é essencial para a realização de uma vida plena20.

Quanto aos itens que obtiveram valores considerados menores, estão os componentes atividade física e nutrição (Tabela 1). 0 baixo índice obtido em atividade física e nutrição pode estar relacionado com a demanda de carga horária desenvolvidas pelos policiais ou ainda pela instabilidade de horários fixos para a prática de atividade física e sua alimentação no horário de trabalho8. Neste sentindo, entende-se que enquanto está em período de serviço, o policial não consegue realizar as refeições de acordo com as recomendações nutricionais, muito menos selecionar os alimentos apropriados para uma alimentação saudável, visto que muitas vezes as horas de se alimentar não são respeitas em virtude do atendimento de diversas ocorrências ao longo da jornada de trabalho21. E quanto à prática de atividade física, o policial passa exercendo sua função nas ruas, como por exemplo, em uma escala de 12 horas, o tempo para realizar uma atividade física fica prejudicado pelo cansaço físico e mental, preferindo este muitas vezes o descanso. No entanto a atividade física influência de maneira positiva a saúde física e psicossocial, sendo importante em todos os estágios da vida, pois atua de maneira positiva no estado de espirito, na ansiedade, na depressão e no estresse psicológico, além disso é capaz de melhorar a função cognitiva22.

Observa-se que os policiais obtiveram um baixo valor referente ao controle de estresse (Tabela 1). Tal achado pode estar relacionado ao sofrimento físico e mental resultante do conjunto de situações vivenciadas no cotidiano do trabalho, tais como a excessiva jornada de trabalho, o pouco tempo para descanso e lazer, as precárias condições materiais e técnicas e baixos salários8,23. Uma vez que o estresse é caracterizado como a capacidade de adaptação do indivíduo frente a um novo desafio, isto é, que pode deixar o organismo em tensão e inquietude, não só a nível de suas forças físicas, mas principalmente psíquicas, compreende-se que o cargo de policial exige a adaptação do soldado frente a diversos desafios enfrentados no cotidiano, causando esse sentimento de estresse24.

Quando comparado entre sexo, os policiais do sexo feminino apresentaram maior percepção de estilo de vida nos componentes Nutricional $(p=0,001)$, Atividade Física $(p=0,001)$, Relacionamento Social $(p=0,001)$ e Controle do Estresse $(p=0,001)$ em comparação aos policiais do sexo masculino (Tabela 2). Frente à falta de estudos que comparam a qualidade de vida entre policiais homens e mulheres, podemos remeter essa diferença ao verificamos que o corpo da mulher tem sido mais controlado e sujeito a uma pressão social maior relativamente ao corpo do homem, o que reflete de um processo histórico25. As mulheres são mais cuidadosas com as questões que remetem a beleza física, assim adotam rotinas que equilibram atividade física com alimentos saudáveis. Isso pode influenciar no maior relacionamento social, visto que, as mulheres acabam se inserindo em grupos sociais, como de academias, ginásticas, grupos de corrida, entre outros, e com o exercício reflete na diminuição dos níveis de estresse. Ainda, a mulher exerce papel fundamental em casa quando 0 assunto é saúde, pois é dela o papel de cuidadora da família. Enquanto as mulheres preocupam-se com a aparência, peso e com o envelhecimento bem-sucedido, as pesquisas revelam que a maioria dos homens se considera imune às doenças, priorizando os prazeres da vida em detrimento à saúde25.

Outro fator pode estar relacionado a função que a mulher exerce dentro da polícia militar, uma vez que os trabalhos administrativos são mais voltados para esse público, e este por sua vez possui a carga horária mais flexível, aproveitando melhor o tempo livre para cuidados com a saúde, como, prática regular de atividade física e momentos de lazer26-27.

Embora haja uma ampla discussão sobre masculinidade na área da saúde em geral, ainda há uma insuficiência 
de estudos sobre o empenho masculino voltado para o estilo de vida saudável e a promoção da saúde. Para se avançar nessa discussão, dentre outros aspectos, é importante dar voz aos próprios homens para melhor compreender as questões envolvidas no seu acesso aos serviços de saúde28.

Frente aos resultados obtidos na correlação (Tabela 3), observou-se em comum para ambos os sexos que as correlações que mais se destacaram foram entre atividade física com nutricional e atividade física com controle de estresse. Estas correlações reforçam a importância de se praticar atividade física, uma vez que está ligada diretamente com a melhoria da eficiência do metabolismo e diminuição do estresse. Estudos ainda comprovam a respeito dos efeitos e benefícios do exercício físico sobre a saúde do ser humano29-30 e apontam benefícios tais como redução nos níveis de estresse e depressão, aumento do bem-estar físico e psicológico, melhor funcionamento orgânico geral, disposição física e mental, maior rendimento no trabalho entre outros.

Apesar de não terem sido encontrados níveis satisfatórios para atividade física e nutricional para a presente população, ressalta-se a importância e sugere-se que os policiais se atentam aos cuidados com seus hábitos alimentares e prática de atividade física, sendo estas, essenciais para uma boa qualidade de vida e melhor rendimento no trabalho.

Apesar das contribuições dos achados desta pesquisa para a literatura, limitações necessitam ser destacadas. Primeiramente, em relação à abrangência do estudo que esteve restrito ao município de Maringá, impossibilitando a generalização dos resultados obtidos para o contexto brasileiro. No entanto, foram avaliados policiais de diferentes regiões do município, proporcionando assim um panorama do estilo de vida dos policiais que trabalham em prol da segurança do município. Outra limitação refere-se à análise levando em consideração aos cargos dos policiais. Dessa forma, futuras pesquisas devem continuar a explorar o estilo de vida de policiais de diferentes regiões do estado e até mesmo a nível nacional, levando em consideração o cargo desenvolvido (policiais operacionais e administrativos) e outras variáveis que possam ser relacionadas ao estilo de vida dos mesmos com intuito de analisar se há diferenças significativas entre os dois grupos.

Este estudo apresenta implicações práticas relevantes para essa população, destacando a conscientização de que o estilo de vida irá refletir na saúde e na qualidade de vida dos policiais. Neste sentido é importante que os policiais tenham conhecimento que os componentes relacionados ao estilo de vida listados no presente estudo vão influenciar na sua saúde e também no seu rendimento no trabalho. É importante que os oficiais e dirigentes dos batalhões tenham tal conhecimento sobre essas variáveis, a fim de oportunizar atividades no ambiente de trabalho, como ginástica laboral, convênios com academias para os servidores. $E$ ainda criar uma equipe de monitoramento de saúde com nutricionista, profissional de educação física, médico, psicólogo e campanhas preventivas e educativas sobre a importância de manter hábitos saudáveis para a conscientização sobre o estilo de vida.

\section{Considerações Finais}

Frente aos resultados, pode-se concluir que os policiais militares avaliados apresentaram satisfatório estilo de vida com destaque para os componentes Comportamento Preventivo e Relacionamento Social. Por outro lado, nota-se que os componentes Nutricional e Atividade Física de forma geral podem ser melhorados, visto que ainda existem policiais não praticantes de atividades físicas e muitos que não possuem hábitos alimentares saudáveis. $E$ ainda se constatou que 0 componente Controle de Estresse obteve o índice mais baixo, provavelmente gerado pelo desgaste físico e emocional no qual os policiais são submetidos na profissão. As mulheres quando comparadas aos homens demonstram melhores resultados nos componentes nutricional, atividade física, comportamento preventivo e relacionamento Social, mostrandose mais perceptivas ao estivo de vida saudável. Evidenciou-se também a importância da atividade física, uma vez que este componente esteve relacionado com a nutrição e controle do estresse em ambos os sexos.

\section{Referências}

1 Souza Filho MJD, Noce F, Andrade AGPD, Calixto, RDM, Albuquerque, MR, Costa, VT. (2015). Avaliação da qualidade de vida de policiais militares por meio do instrumento WHOQOL-Bref. Revista Brasileira de Ciência e Movimento
23(4):159-169

2 Benevides-Pereira AMT. Burnout: quando o trabalho ameaça o bem-estar do trabalhador. São Paulo: Casa do Psicólogo; 2010.

3 Souza ER, Minayo MCS, Silva JG, Pires TO. Fatores associados ao sofrimento psíquico de policiais militares da cidade do Rio de Janeiro, Brasil. Cad Saude Publica 2012; 28(7):1297-311.

4 Rodrigues CF. Perfil do estilo de vida dos profissionais da segurança pública de uma delegacia de Polícia Civil [Monografia]. Academia de Polícia Militar Costa Verde. Porto velho, 2008.

5 Souza ERD, Minayo MCDS, Silva JG, Pires TDO. Fatores associados ao sofrimento psíquico de policiais militares da cidade do Rio de Janeiro, Brasil. Cad. saúde pública. 2012; 28(7): 1297-1311.

6 Gonçalves BL. Avaliação da aptidão física dos Policiais Militares do CR-II [Monografia]. Academia de Polícia Militar Costa Verde. Várzea Grande, 2012.

7 Smith TC, Zamorski M, Smith B, Riddle JR, LeardMann CA, Wells TS. The physical and mental health of a large military cohort: baseline functional health status of the Millennium Cohort. BMC Public Health 2007; 7, 340.

8 Jesus MJ, Jesus AFEA. Nível de atividade física e barreiras percebidas para a prática de atividades físicas entre Policiais Militares. Revista Brasileira de Ciência da Esporte. 2012, 34(2): 433-448.

9 Silva R, Schlichting AM, Schlichting JP, Gutierres Filho PJ, Adami F, Silva A. Aspetos relacionados à qualidade de vida e atividade física de policiais militares de Santa Catarina-Brasil. Motricidade. 2012, 8(3): 81-89.

10 Wagner LC, Stankievich RAP, Pedroso F. Saúde mental e qualidade de vida de policiais civis da região metropolitana de Porto Alegre. Rev Bras Med Trab. 2012, 10(2): 64-71.

11 da Silva EFC, Hernandez MSSS, Gonçalves EE, da Silva Castro TL, Beatriz E, Arancibia AV, da Silva PR. Qualidade de vida de policiais: uma revisão sistemática de estudos observacionais. Revista Cubana de Medicina Militar. 2014, 43(3): $341-351$

12 Lima Filho DP, Cassundé FRDSA. Uma Perspectiva sobre as condições de trabalho e qualidade de vida dos policiais da 25a. CIPM na cidade de casa Nova no Estado da Bahia. Revista De Psicologia. 2015, 9(25):139-158.

13 Nahas MV; Barros M. VG; Francalacci VL. O pentáculo do bem-estar: base conceitual para avaliação do estilo de vida dos individuos ou grupos. Revista Brasileira de Atividade Física e Saúde. 2000, 5(2):48- 59.

14 Nahas MV, de Barros MV, Francalacci V. O pentáculo do bem-estar-base conceitual para avaliação do estilo de vida de indivíduos ou grupos. Revista Brasileira de Atividade Física \& Saúde. 2012, 5(2):48-59.

15 Bittner E. Aspectos do trabalho policial. São Paulo: Editora da Universidade de São Paulo; 2003.

16 Nahas MV. Atividade física, saúde e qualidade de vida: conceitos e sugestões para um estilo de vida ativo. 4ed. Londrina: Midiograf; 2006.

17 Kloster SH, Prati SRA. Análise do estilo de vida de estudantes universitários - um estudo transversal. Revista de Educação Física/UEM. 2007, 18:247-250. 
18 Nahas MV. Atividade Física, Saúde e Qualidade de Vida. Conceitos e Sugestões para um Estilo de Vida Ativo. Midiograf, Londrina; 2001.

19 Minayo MCS, Souza ER, Constantino P. Riscos percebidos e vitimização de policiais civis e militares na segurança pública. Cadernos de Saúde Pública. 2007, 23(11).

20 Gonçalves A, Vilarta R. Qualidade de vida e atividade física - Explorando teoria e prática. São Paulo: Manole; 2004.

21 Minayo MCS, Assis, SG, Oliveira RVC. Impacto das atividades profissionais na saúde física e mental dos policiais civis e militares do Rio de Janeiro (RJ, Brasil). Ciência e Saúde Coletiva. 2011, 16(4):2199-2209.

22 Allsen PE, Harrioson JM, Vance B. Exercício e Qualidade de vida - Uma abordagem personalizada. 6 ed. São Paulo: Manole; 2001.

23 Costa M, Júnior AH, Oliveira J, Maia E. Estresse: diagnóstico dos policiais militares em uma cidade brasileira. Revista Panamericana de Salud Pública. 2007, 21, 4:217-222.

24 Bacarro A. Vencendo o stress: como detecta-lo e supera-lo. Ed. Vozes. Petrópolis, Rio de Janeiro; 1997.

25 Torres MCC, Silva P, Novais C, Carvalho J. Gênero, sexualidade e atividade física: uma leitura sobre masculinidades e feminilidades (re) construídas à luz do envelhecer. Revista Brasileira de Ciências do Envelhecimento Humano. 2013, $9(1)$.

26 Luz ES. Estudo do Estresse Ocupacional em Mulheres. Policiais Militares da Cidade do Rio de Janeiro [dissertação]. Rio de Janeiro (RJ): Instituto Fernandes Filgueira, Fundação Oswaldo Cruz; 2011.

27 Soares BM, Musumeci L. Mulheres Policiais: presença feminina na Polícia Militar do Rio de Janeiro. Rio de Janeiro: Civilização Brasileira; 2005.

28 Gomes R, Nascimento, EF, Araujo FC. Por que os homens buscam menos os serviços de saúde do que as mulheres? As explicações de homens com baixa escolaridade e homens com ensino superior. Caderno de Saúde Pública. 2007, 23(3):565-574.

29 Salmuski DM, Noce F. Importância da atividade física para a saúde e qualidade de vida: um estudo entre professores, alunos e funcionários da UFMG. Revista Brasileira de Atividade Física e Saúde. 2000, 5(1):5-21.

30 Macedo CDSG, Garavello JJ, Oku EC, Miyagusuku FH, Agnoll PD, Nocetti PM. Benefícios do exercício físico para a qualidade de vida. Revista Brasileira de Atividade Física \& Saúde. 2012, 8(2):19-27.

\section{AGRADECIMENTOS}

José Roberto Andrade do Nascimento Junior e Leidyane Aparecida De Souza Amoroso de Souza colaboraram com a concepção do projeto, análise e interpretação dos dados. Gislaine Contessoto Pizzo colaborou com a redação do artigo. Daniel Vicentini de Oliveira e Lenamar Fiorese Vieira colaboraram com a revisão e aprovação final da versão a ser publicada. 